Open Access

Original Article

\title{
Post-ERCP Pancreatitis: Risk factors and role of NSAIDs in primary prophylaxis
}

\author{
Muhammad Haseeb Nawaz', \\ Shahid Sarwar ${ }^{2}$, Muhammad Arif Nadeem ${ }^{3}$
}

\begin{abstract}
Objective: To determine efficacy of diclofenac suppository in reducing post-ERCP pancreatitis (PEP) and identify risk factors for PEP.

Methods: This is a placebo-based prospective study at Department of Medicine \& Gastroenterology, Services Institute of Medical Sciences / Services Hospital, Lahore performed from January 2018 to June 2019. Patients were randomized to receive diclofenac suppository or glycerine suppository before ERCP. Both groups were compared for PEP using chi square $x^{2}$ test while risk factors for PEP were determined using binary logistic regression.

Results: Total of 165 patients with mean age $49.1( \pm 15.2)$ and male to female ratio $1 / 1.6(63 / 102)$ were included. Among $82(49.7 \%)$ patients in diclofenac group, 8 (9.7\%) developed pancreatitis while $19(22.9 \%)$ of $83(50.3 \%$ ) in placebo group had PEP ( $\mathrm{p}$ value 0.02 ). After multivariate analysis, age $>45$ years ( $\mathrm{p}$ value 0.014 , OR 3.2), Bilirubin $>3 \mathrm{mg} / \mathrm{dl}$ ( $\mathrm{p}$ value $0.004 \mathrm{OR} 3.58$ ), time to cannulation $>5$ minutes ( $\mathrm{p}$ value $<0.000$ OR 9.2), use of precut ( $p$ value $<0.000$ OR 4.9), pancreatic duct cannulation ( $p$ value 0.000 OR 5.46) and total procedure time $>30$ minutes ( $p$ value 0.01 OR 3.92) were risk factors for PEP.

Conclusion: Pre-procedure Diclofenac suppository reduces post-ERCP pancreatitis. Age $>45$ years, serum bilirubin $>3 \mathrm{mg} / \mathrm{dl}$, cannulation time $>5$ minutes, use of precut, pancreatic duct cannulation and procedure time $>30$ minutes are risk factors for post-ERCP pancreatitis.
\end{abstract}

KEYWORDS: NSAIDs, Post-ERCP pancreatitis, Primary prophylaxis, Risk factors.

doi: https://doi.org/10.12669/pjms.36.3.1804

How to cite this:

Nawaz MH, Sarwar S, Nadeem MA. Post-ERCP Pancreatitis: Risk factors and role of NSAIDs in primary prophylaxis. Pak J Med Sci. 2020;36(3):426-431. doi: https://doi.org/10.12669/pjms.36.3.1804

This is an Open Access article distributed under the terms of the Creative Commons Attribution License (http://creativecommons.org/licenses/by/3.0), which permits unrestricted use, distribution, and reproduction in any medium, provided the original work is properly cited.

1. Dr. Muhammad Haseeb Nawaz, MBBS,

Post Graduate Resident,

2. Dr. Shahid Sarwar, MBBS, FCPS (Med), FCPS (Gastroenterol), MCPS-HPE, FRCP (Edin)

Associate Professor,

3. Prof. Dr. Muhammad Arif Nadeem MBBS, FCPS (Medicine)

1-3: Medical Unit-III,

Department of Medicine \& Gastroenterology,

Services Institute of Medical Sciences,

Lahore, Pakistan.

Correspondence:

Dr. Shahid Sarwar,

Associate Professor,

Services Institute of Medical Sciences,

Lahore, Pakistan.

Address: 153-D, Muslim Road, Jinnah Colony,

Samanabad, Lahore, Pakistan.

Email: shahidsarwardr@gmail.com

* Received for Publication:

October 11, 2019

* Accepted for Publication:

January 2, 2020

\section{INTRODUCTION}

Endoscopic Retrograde Cholangiopancreatography (ERCP), since its inception in 1968 has revolutionized the management of pancreatico-biliary diseases. ${ }^{1}$ It allows access to biliary and pancreatic ducts for diagnosis as well as management without risks of prolonged anesthesia, surgical trauma, surgical complications and extended hospital stay for post-operative recovery with technically difficult pancreatico-biliary surgeries. ${ }^{2}$

In last few decades, with availability of sophisticated new accessories of ERCP, range of therapeutic interventions possible in ERCP has remarkably extended. However, this has resulted in increasing incidence of complications like post ERCP pancreatitis, cholangitis, iatrogenic bleeding, sepsis, perforation etc. ${ }^{3}$ Complications 
like hypoxia, aspiration pneumonia and cardiopulmonary depression are increasing due to extended anesthesia time during these therapeutic interventions. ${ }^{4}$

Among all potential complications of ERCP, post ERCP pancreatitis (PEP) is a common complication associated with significant morbidity and mortality. ${ }^{5}$ Its incidence varies between 1-40\% depending on patient related co-morbidities, type of intervention, duration of procedure and expertise of endoscopist. ${ }^{6}$ PEP is mostly selflimiting responding to conservative treatment but still caries mortality of $0.7 \%{ }^{6}$

Post ERCP pancreatitis is the result of mechanical, thermal, chemical, enzymatic or hydrostatic injury inflicted during procedure. Based on this pathophysiology, different interventions have been tried for prophylaxis of PEP. ${ }^{7}$ It includes ensuring pancreatic drainage via stenting, inhibition of intra-acinar trypsinogen activation via protease inhibitors, reducing sphincter of oddi spasm with glucagon or glyceryl trinitrate (GTN) and facilitation of cannulation with secretin injection. ${ }^{8}$ However most promising results are shown by anti-inflammatory drugs targeting chemical injury induced cascade of inflammation. ${ }^{9}$

Non-steroidal anti-inflammatory drugs (NSAIDs) are inexpensive, easily administered and effective inhibitors of phospholipase A2 and cyclooxygenase which can block inflammation leading to acute pancreatitis. ${ }^{9}$ Anti-inflammatory drugs evaluated for their efficacy in preventing PEP include indomethacin, celecoxib and diclofenac sodium with mixed results. In a study of 602 patients by Elmunzer et al, reduction in incidence of PEP from $16.9 \%$ to $9.2 \%$ was noted with use of indomethacin. ${ }^{10}$ Otsuka et al. noted reduction in PEP from $18.9 \%$ to $3.9 \%$ with use of rectal diclofenac suppository before ERCP. ${ }^{11}$ However no benefit of administering indomethacin in controlling PEP was seen in a study of 665 patients by Dobronte et al. ${ }^{12}$ It is due to these conflicting results that despite recommendation by European Society of Gastrointestinal Endoscopy (ESGE) to use NSAIDs for $\mathrm{PEP}^{13}$, its role is still under intense debate. We planned a placebo based, case control study to determine efficacy of NSAIDs (Diclofenac) suppository in preventing post ERCP pancreatitis and to identify risk factors predisposing to PEP.

\section{METHODS}

A quasi-Experimental, placebo based case control, triple blind study was carried out at
Department of Medicine \& Gastroenterology, Services Institute of Medical Sciences / Services Hospital, Lahore from January 2018 to June 2019 after the approval of Internal Review Board (Ref No. IRB/2018/464/SIMS, dated Sept. 25, 2018). All patients aged above 18 years being admitted for ERCP willing to participate in study were included after informed consent. Patients with allergy to NSAIDs, contraindication for NSAIDs use (i.e. active peptic ulcer disease, serum creatinine $>1.4 \mathrm{mg} / \mathrm{dl}$ ), history of pancreatitis within last 4 weeks, use of NSAIDs in preceding two weeks, antibiotic use within 4 weeks and pregnant and nursing mothers were excluded from study.

Detailed clinical interview regarding symptoms, indication for procedure and co-morbid issue followed by clinical examination was carried out. Laboratory and radiological investigation results including complete blood count, liver function tests, renal function tests, abdominal ultrasound, CT scan, MRI or MRCP if performed were recorded. Patients were randomized in two groups using online random table generator stat trek ${ }^{\circledR}$. Patients of Group-A were given Diclofenac sodium suppository by nursing assistant at least 15 minutes before procedure while Group-B patient received Glycerine suppository as placebo. Identity of patient group was not known to patient, endoscopist and team responsible for patient follow up after procedure.

All ERCP procedures were performed by two senior endoscopists, under Propofol sedation by a dedicated senior nursing assistant with continuous monitoring of vital signs. All maneuvers and interventions done during ERCP including time of biliary cannulation which was always wire guided, use of needle knife sphincterotomy, pancreatic duct cannulation/contrast injection, sphincterotomy/sphincteroplasty, balloon sweep for stone extraction, biliary or pancreatic stenting and total duration of procedure were recorded.

After ERCP, patients were kept in high dependency unit (HDU) for at least 24 hours. Patients were monitored for new onset abdominal symptoms including pain, vomiting, distention or absolute constipation. Serum amylase/lipase were checked at six hours and 24 hours' postprocedure. Primary study end point was postERCP pancreatitis which was defined and staged according to Atlanta criteria which defines PEP as presence of at least 2 of 3 features including; 
(1) Abdominal pain consistent with acute pancreatitis

(2) At least 3 times increase in serum amylase or lipase

(3) Evidence of pancreatic inflammation on abdominal ultrasound, CT scan or MRI. ${ }^{14}$

Diclofenac sodium was considered effective if incidence of PEP decline by $>50 \%$. Secondary end points were risk factors associated with PEP. Post procedure follow up was done by senior team members, unaware of treatment given as prophylaxis for PEP. Patients with PEP were managed as per standard protocols for managing acute pancreatitis.

Statistical Analysis: We estimated that a sample size of 164 will give $80 \%$ power to detect at least $50 \%$ reduction in incidence of PEP (i.e. from $20 \%$ to $10 \%$ ) with $5 \%$ margin of error. Data was analyzed using SPSS $22^{\circledR}$ (Armonk NY: IBM corp.) by statistician unaware of drugs used in two groups. Quantitative variables with normal distribution were expressed as mean \pm standard deviation (SD), nonparametric variables were given as median \pm interquartile range (IR) whereas qualitative variables were given as percentage. Primary and secondary outcome variables were compared between two groups using unpaired student's $t$ test and chi square $\left(x^{2}\right)$ test to determine Odd's ratio (OR) for PEP and Mann Whitney $U$ test for non-parametric variables.

Cut off values for numerical variables for predicting PEP were determined using Receiver operating characteristic (ROC) curve identifying coordinate point with best sensitivity and specificity. A multi-variate binary logistic regression analysis was performed for variables with statistical significance on uni-variate analysis $(p \leq 0.05)$ using post-ERCP pancreatitis as dependent variable. Predictive value of model was checked by determining two log likelihood and testing with Hosmer and Lemeshow test. P value of less than 0.05 was considered statistically significant.

\section{RESULTS}

Total of 165 patients were included with mean age of $49.1( \pm 15.2)$ and male/female ratio of $1 / 1.6$ (63/102). Predominant presenting complaints in patients included were abdominal pain 131 (79.4\%), jaundice 101 (61.2\%), fever $79(47.9 \%)$, itching $78(47.3 \%)$ and weight loss in $66(40 \%)$ patients. Previous history of cholecystectomy was present in $28(17 \%)$ patients, who were undergoing ERCP for residual stones $21(75 \%)$ and iatrogenic
CBD injury seven (25\%). Diabetes mellitus was present in $32(19.4 \%)$ patients while $43(26.1 \%)$ were hypertensive.

Majority of patients were being treated for common bile duct (CBD) stones 97 (58.8\%) whereas pancreatic carcinoma 17 (10.3\%), cholangiocarcinoma $11(6.7 \%)$, periampullary cancer $11(6.7 \%)$, gall bladder CA seven $(4.2 \%)$ and CBD leakage $10(6.1 \%)$ were other major indications for ERCP.

Randomization lead to $82(49.6 \%)$ patients in study group receiving Diclofenac suppository before procedure while $83(50.4 \%)$ patients in placebo group, treated with glycerine suppository. We compared both groups for baseline variables as shown in Table-I.

During ERCP, cannulation was achieved within five minutes in $89(53.9 \%)$ patient, needle knife sphincterotomy (precut) was needed in 28(17\%) patients while 131 (47.3\%) had routine sphincterotomy after cannulation. Pancreatic duct (PD) was cannulated in $45(27.3 \%)$ patients and 10 (6.1\%) had contrast injection in PD as well. CBD stones were extracted in $83(50.3 \%)$ patients while $78(47.3 \%)$ had biliary stenting and $10(6.1 \%)$ had CBD dilatation.

After ERCP, 57 (34.5\%) patients complained of abdominal pain, 25 (15.2\%) had vomiting and $16(9.7 \%)$ felt abdominal distension. These complaints settled in majority of these patients within few hours. Post-ERCP pancreatitis (PEP) was diagnosed in $27(16.4 \%)$ patients, all of whom had mild pancreatitis and recovered in few days without necrosis or multi-organ failure. Asymptomatic hyper-amylasemia was seen in $13(7.9 \%)$ patients. PEP was significantly more in placebo group $19(22.9 \%)$ patients as compared to eight $(9.7 \%)$ patients in study group receiving diclofenac suppository ( $\mathrm{p}$ value 0.02 ) confirming its efficacy with $>50 \%$ reduction in PEP with Odds ratio (OR) of 0.36 (95\% CI: $0.14-0.88)$ in favor of diclofenac group.

On uni-variate analysis of variables for its association with PEP, we identified age $>45$ years ( $\mathrm{p}$ value 0.014 , OR 3.2 95\% CI:1.2-8.4), Bilirubin $>3 \mathrm{mg} / \mathrm{dl}$ ( $\mathrm{p}$ value 0.004 , OR 3.58 95\% CI: 1.48.7 ), time of cannulation(TTC) $>5$ minutes ( $\mathrm{p}$ value $<0.000$, OR $9.295 \% \mathrm{CI}: 3.0-28.1)$, use of needle knife for cannulation(precut) ( $\mathrm{p}$ value $<0.000$, OR 4.9 95\% CI: 1.9-12.2), pancreatic duct cannulation (p-value<0.000, OR 5.46 95\% CI: 2.2-13) and total procedure time $($ TPT) $>30$ minutes $(\mathrm{p}$ value 0.01 , 


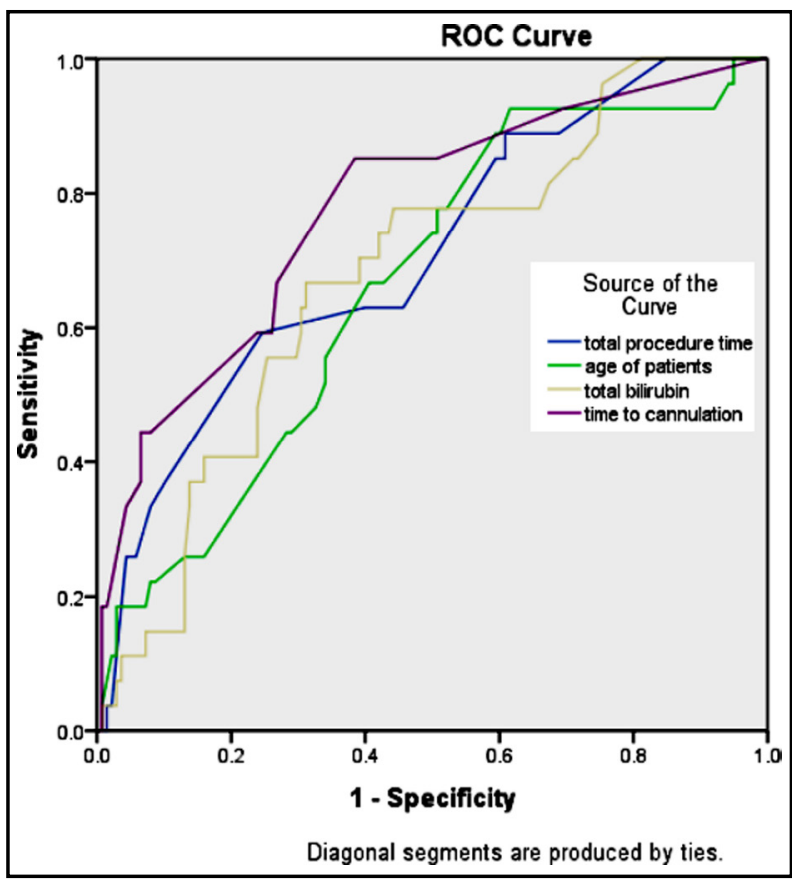

Fig.1: ROC Curve to identify best coordinate points.

OR 3.92 95\% CI:1.2-11.9) as significant risk factors for PEP. However, patients undergoing ERCP for CBD stones had significantly less chance of developing PEP ( $p$ value 0.003, OR 0.28 95\% CI 0.11-0.68). ROC curves to identify cut off values of age, bilirubin, TTC and TPT are shown in Fig.1.

\section{Area Under the Curve}

Test Result Variable(s) Area

Total procedure time $\quad 0.709$

Age of patients $\quad 0.664$

Total bilirubin $\quad 0.681$

Time to cannulation $\quad 0.777$
Table-II: Hosmer and Lameshow goodness of fit testing of model for predicting PEP.

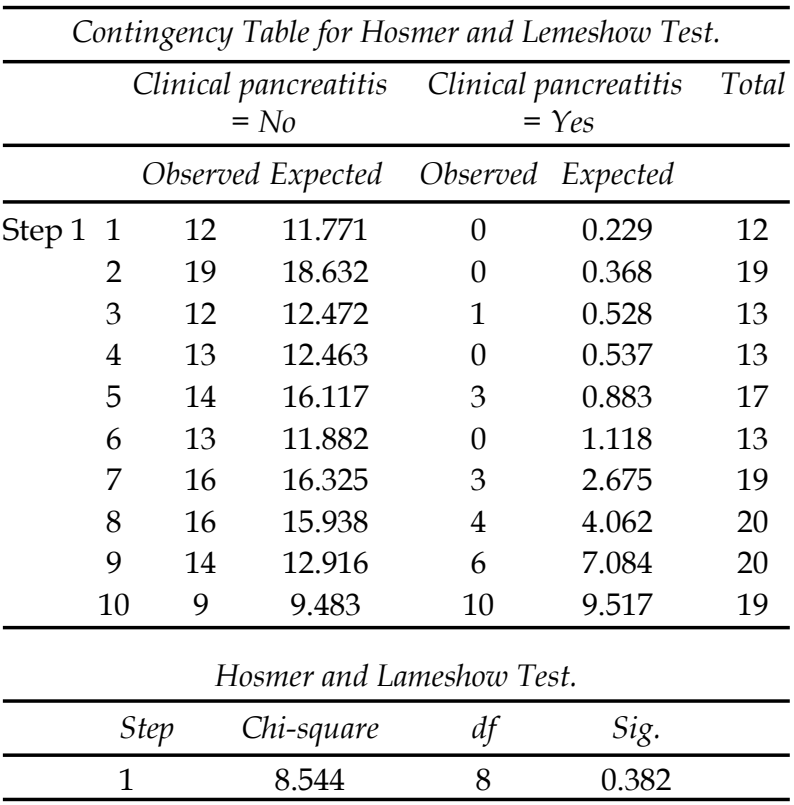

The test result variable(s): total procedure time, age of patients, total bilirubin and time to cannulation has at least one tie between the positive actual state group and the negative actual state group. Statistics may be biased.

We analyzed prediction model comprising of age $>45$, serum bilirubin $>3 \mathrm{mg} / \mathrm{dl}$, time to cannulation (TTC) $>5$ minutes, precut, pancreatic duct cannulation and total procedure time (TPT)>30 minutes via binary logistic regression. Our model accurately predicted chances of PEP in $86.1 \%$ cases ( $\mathrm{p}$-value of $<0.000$ ) with 2 log likelihood ratio of 114.87. Hosmer and Lameshow goodness of fit testing revealed no difference in model based expected outcome and observed outcome ( $\mathrm{p}$-value 0.38) as shown in Table-II and Table-III.

Table-I: Comparison of patients in study group and placebo group.

\begin{tabular}{lccc}
\hline Variable & $\begin{array}{c}\text { Study group (Diclofenac } \\
\text { suppository) }(n-82)\end{array}$ & $\begin{array}{c}\text { Placebo group (Glycerine } \\
\text { Suppository) }(n-83)\end{array}$ & $\begin{array}{c}\text { P-value } \\
\text { Age (mean years } \pm \text { SD) }\end{array}$ \\
Duration of illness (median $\pm \mathrm{IQR})$ & $47.7 \pm(14.8)$ & $50.2 \pm(15.5)$ & 0.28 \\
Serum bilirubin (median $\pm \mathrm{IQR})$ & $122 \pm(189)$ & $116 \pm(159)$ & $0.81^{*}$ \\
Male/Female (number) & $7 \pm(8)$ & $35 / 4)$ & $0.15^{*}$ \\
H/O Surgery & $28 / 54$ & 13 & 0.28 \\
Diabetes mellitus & 15 & 17 & 0.65 \\
Hypertension & 15 & 22 & 0.72 \\
CBD stone & 21 & 46 & 0.89 \\
Malignancy Cholangio/pancreatic/Gall bladder & $8 / 21 / 6$ & $14 / 17 / 1$ & 0.37 \\
CBD leakage & 5 & 5 & 0.12 \\
\hline
\end{tabular}

*Mann Whittney U-test, IQR: Interquartile Range. 
Role of NSAIDs in post-ERCP pancreatitis

Table-III: Classification Tablea.

\begin{tabular}{|c|c|c|c|c|c|}
\hline \multicolumn{3}{|c|}{ Observed } & \multicolumn{3}{|c|}{ Predicted } \\
\hline & & & \multicolumn{2}{|c|}{ Clinical pancreatitis } & \multirow[t]{2}{*}{ Percentage Correct } \\
\hline & & & No & Yes & \\
\hline \multirow[t]{3}{*}{ Step 1} & Clinical pancreatitis & No & 134 & 4 & 97.1 \\
\hline & & Yes & 19 & 8 & 29.6 \\
\hline & Overall Percentage & & & & 86.1 \\
\hline
\end{tabular}

\section{DISCUSSION}

Despite lot of technological advances in endoscopy, post-ERCP pancreatitis continues to be a major complication encountered with incidence varying from $1 \%$ to $40 \%$ depending on patient as well as procedure related risk factors. Syren E et al. in a retrospective analysis identified female gender, age below 65 years and hyperlipidemia as potential risk factors for PEP. ${ }^{15}$ More than 10 attempts at cannulation (OR 14.9), previous PEP (OR 8.7), precut (OR 3.1), pancreatic duct cannulation (OR 2.1) were identified as risk factor for PEP in a prospective multicenter study. ${ }^{16}$ Li GZ et al in a study of 1786 ERCPs, noted 3.8\% incidence of PEP and identified pancreatic deep wire pass, metal biliary endoprosthesis, post liver transplantation and post-fistulotomy ERCP as risk factors of PEP. ${ }^{17}$ Suspected sphincter of Oddi dysfunction, presence of hilar obstruction, number of cannulation attempts $>13$, pancreatic duct cannulation $\geq 1$ and pancreatic contrast injections $\geq 1$ were potential risk factors for PEP in a study of 790 patients by Kang $X$ et al. ${ }^{18}$ Difficult and prolonged cannulation ( $\mathrm{p}$ 0.002), pancreatic duct cannulation (p 0.001) and pancreatic duct contrast injection $(p<0.001)$ were associated with PEP in a study from Karachi. ${ }^{19}$

We in our study identified two patient related risk factors, age $>45$ years and bilirubin $>3 \mathrm{mg} /$ $\mathrm{dl}$ and four procedure related factors time taken for cannulation $>5$ minutes, use of needle knife, pancreatic duct cannulation and procedure time $>30$ minutes to be associated with postERCP pancreatitis. Prolonged cannulation time, pancreatic duct cannulation and needle knife use results in mechanical and thermal injury increasing risk of inducing cascade of pancreatic inflammation. Synergy of these risk factors increases chances of $\mathrm{PEP}^{13}$ as verified by $86.4 \%$ accuracy in predicting PEP in our study when these risk factors are combined. Due care for indication of procedure, improved technical skills with avoidance of hazardous interventions like pre-cut or unintended pancreatic duct cannulation and efficient procedure time can reduce chances of PEP.

We diagnosed PEP in $16.4 \%$ study patients, significantly less $(9.7 \%)$ in study group treated with Diclofenac suppository before procedure than placebo group $(22.9 \%)$. Out of all interventions tried to avoid PEP, NSAIDs use has shown best results. In a meta-analysis of 19 RCT involving 5031 patients NSAIDs use was associated with significant PEP risk reduction ( $R R=0.45,95 \%$ ci 0.30 to 0.67$).{ }^{20}$ Serrano JPR did a systematic review of 21 RCTs comprising of 6854 patients comparing NSAIDs vs placebo before ERCP and concluded that only rectal administration reduces incidence of PEP (6.8\% VS 13\%; 95\% CI 0.10-0.04, Numbers needed to treat (NNT) 20, P <0.05). Moreover, only diclofenac and indomethacin were effective in preventing PEP. ${ }^{21}$ It is due to this robust evidence that ESGE has recommended routine use of rectal NSAIDs before every ERCP. ${ }^{13}$

Despite recent recommendation by ESGE, use of NSAIDs for PEP prophylaxis is not common in clinical practice. In a recent survey from Portugal, only $54 \%$ patients undergoing ERCP received rectal NSAIDs. ${ }^{22}$ Similarly $64.1 \%$ of PEP prophylaxis non users cited lack of conviction in its benefit for their decision in a survey from UK. ${ }^{23}$ Studies like ours depicting clear benefit of using NSAIDs for preventing PEP will promote its use in clinical practice leading to significant reduction in potentially lethal complication like PEP.

\section{CONCLUSION}

Pre-procedure Diclofenac suppository reduces post-ERCP pancreatitis. Age $>45$ years, serum bilirubin $>3 \mathrm{mg} / \mathrm{dl}$, cannulation time in excess of 5 minutes, use of precut, pancreatic duct cannulation and procedure time $>30$ minutes are risk factors for post-ERCP pancreatitis.

Grant support \& Financial support: None. 


\section{REFERENCES}

1. McCune WS, Shorb PE, Moscovitz H. Endoscopic cannulation of the ampulla of vater: a preliminary report. Ann Surg. 1968;167(5):752-756. doi: 10.1097/00000658196805000-00013

2. Matsushita M, Koyabu M, Nishio A, Seki T, Okazaki K. Techniques of ERCP with a conventional endoscope in pancreatoduodenectomy anatomy. Gastrointest Endosc. 2017;86:747-748. doi: 10.1016/j.gie.2017.05.007

3. Guda NM, Freeman ML. Overview of ERCP Complications: Prevention and Management. Lee LS, editor ERCP and EUS: A Case-Based Approach. New York: Springer. 2015;37-56. doi: 10.1007/978-1-4939-2320-5

4. Kahaleh M, Freeman M. Prevention and management of post-endoscopic retrograde cholangiopancreatography complications. Clin Endosc 2012;45:305-312. doi: $10.5946 /$ ce.2012.45.3.305

5. Mine T, Morizane T, Kawaguchi Y, Akashi R, Hanada $\mathrm{K}$, Ito $\mathrm{T}$, et al. Clinical practice guideline for postERCP pancreatitis. J Gastroenterol. 2017;52:1013-1022. doi: 10.1007/s00535-017-1359-5

6. Zhou W, Li Y, Zhang Q, Li X, Meng W, Zhang L, et al. Risk factors for postendoscopic retrograde cholangiopancreatography pancreatitis: A retrospective analysis of 7,168 cases. Pancreatology. 2011;11(4):399-405. doi: 10.1016/S1424-3903(11)80094-3

7. Freeman ML, Guda NM. Prevention of post-ERCP pancreatitis: a comprehensive review. Gastrointest Endosc. 2004;59:845-864. doi: 10.1016/S0016-5107(04)00353-0

8. Zhang H, Cho J, Buxbaum J. Update on the Prevention of Post-ERCP Pancreatitis. Curr Treat Options Gastroenterol. 2018;16:428-440. doi: 10.1007/s11938-018-0194-y

9. Andrade-Davila VF, Chavez-Tostado M, Davalos-Cobián C, Garcia-Correa J, Montano-Loza A, Fuentes-Orozco C, et al. Rectal indomethacin versus placebo to reduce the incidence of pancreatitis after endoscopic retrograde cholangiopancreatography: results of a controlled clinical trial. BMC Gastroenterol. 2015;15:85. doi:10.1186/s12876015-0314-2

10. Elmunzer BJ, Scheiman JM, Lehman GA, Chak A, Mosler P, Higgins PD, et al. U. S. Cooperative for Outcomes Research in Endoscopy (USCORE). A randomized trial of rectal indomethacin to prevent post-ERCP pancreatitis. N Engl J Med. 2012;366:1414-1422. doi:10.1056/NEJMoa1111103

11. Otsuka T, Kawazoe S, Nakashita S, Kamachi S, Oeda S, Sumida C, et al. Low-dose rectal diclofenac for prevention of post-endoscopic retrograde cholangiopancreatography pancreatitis: a randomized controlled trial. J Gastroenterol. 2012;47:912-917. doi: $10.1007 /$ s00535-012-0554-7

12. Dobronte Z, Szepes Z, Izbeki F, Gervain J, Lakatos L, Pecsi $\mathrm{G}$, et al. Is rectal indomethacin effective in preventing of post-endoscopic retrograde cholangiopancreatography pancreatitis? World J Gastroenterol. 2014;20:10151-10157. doi: 10.3748/wjg.v20.i29.10151

13. Dumonceau JM, Andriulli A, Elmunzer J, Mariani A, Meister T, Deviere J, et al. Prophylaxis for Post-ERCP pancreatitis: European Society of Gastrointestinal endoscopy (ESGE) Guidelines- Updated June 2014 Endoscopy. 2014;46:799-815. doi: 10.1055/s-0034-1377875
14. Banks PA, Bollen TL, Dervenis C, Gooszen HG, Johnson $\mathrm{CD}$, Sarr MG, et al. Acute Pancreatitis Classification Working Group. Classification of acute pancreatitis--2012: revision of the Atlanta classification and definitions by international consensus. Gut. 2013;62:102-111. doi: 10.1136/gutjnl-2012-302779

15. Syren E, Eriksson S, Enochsson L, Eklund A, Sandblom G. Risk factors for pancreatitis following endoscopic retrograde cholangiopancreatography. BJS Open. 2019;3(4):485-489. doi: 10.1002/bjs5.50162

16. Testoni PA, Mariani A, Giussani A, Vailati C, Masci E, Macarri $G$ et al. Risk factors for post-ERCP pancreatitis in high and low volume centers and among expert and non expert operators: a prospective multicenter study. Am J Gastroenterol. 2010;105:1753-1761. doi: 10.1038/ajg.2010.136

17. Li GZ, Wand G, Fang J, Zha HL, Zhao Q. Risk factors for post-ERCP pancreatitis: Evidence from 1786 cases. Med Sci Monit. 2018;24:8544-8552. doi: 10.12659/MSM.913314

18. Kang $X$, Zheng L, Zeng W, Yang S, Sun H, Zhang R, et al. Risk factors for Post-ERCP pancreatitis in high risk patients receiving Post-procedure rectal indomethacin. J Gastrointest Surg. 2018;22(11):1903-1910. doi: 10.1007/ s11605-018-3864-0

19. Leghari A, Ghazanfar S, Qureshi S, Taj MA, Niaz SK, Quraishy S. Frequency and risk factors in the post-ERCP pancreatitis in a tertiary care centre. J Coll Physicians Surg Pak. 2013;23(9):620-624. doi: 09.2013/JCPSP.620624

20. Liu L, Li C, Huang Y, Jin H. Nonsteroidal anti-inflammatory drugs for endoscopic retrogradecholangiopancreatography postoperative pancreatitis prevention: a systematic review and meta-analysis. J Gastrointest Surg. 2019;23(10):19912001. doi: 10.1007/s11605-018-3967-7

21. Serrano JPR, de Moura DTH, Bernardo WM, Ribeiro IB, Franzini TP, de Moura ETH, et al. Nonsteroidal antiinflammatory drugs versus placebo for post-endoscopic retrograde cholaniopancreatograaphy pancreatitis: a systematic review and meta-analysis. Endosc Int Open. 2019;7(4): E477-E486. doi: 10.1055/a-0862-0215

22. Lopes L, Canena J. ERCP in Portugal: a wide survey on the prevention of Post-ERCP pancreatitis and papillary cannulation techniques. GE Port J Gastroenterol. 2018;26(1):14-23. doi: 10.1159/000487150

23. Hanna MS, Portal AJ, Dhanda AD, Przemioslo R. UK wide survey on the prevention of post-ERCP pancreatitis. Frontline Gastroenterol. 2014;5(2):103-110.

\section{Author's Contributions:}

MHN: Designed study, data collection, manuscript review.

SS: Conceived and designed study, statistical analysis, manuscript writing, is responsible for integrity of research.

MAN: Designed study, Review and final approval of manuscript. 